\title{
Standardized Allergy Biological Unit per Milliliter
}

National Cancer Institute

\section{Source}

National Cancer Institute. Standardized Allergy Biological Unit per Milliliter. NCI

Thesaurus. Code C116233.

A unit of allergenic product potency expressed as the number of standardized biological units per one milliliter of formulation. 